\title{
AN ADAPTIVE, HIGH-ORDER, NOTCH FILTER USING ALL PASS SECTIONS ${ }^{\dagger}$
}

\author{
Sebastian Torres and Victor DeBrunner \\ The University of Oklahoma \\ School of Electrical and Computer Engineering \\ 202 West Boyd Street, Room 219, Norman, Oklahoma 73019-0631
}

\begin{abstract}
A fully adaptive infinite impulse response notch filter in cascade form is proposed to detect and track multiple time-varying frequencies in additive white noise. Based on transformations for digital filters in the frequency domain, the filter results in a minimal number of parameters. In addition, a simple adaptive algorithm with good tracking and convergence properties is obtained by using all-pass filters and truncating the gradient. Computer simulations are included to verify the competitive performance of this filter under a wide range of conditions. From this analysis, we conclude that our new design is computationally simple, achieves rapid convergence, and is consequently a good choice in many non-stationary environments.
\end{abstract}

\section{INTRODUCTION}

The problem of designing adaptive notch filters (ANF) for retrieving narrow-band signals immersed in broad-band noise has received a great deal of attention since the late 70 's, when the first adaptive line enhancer (ALE) was introduced by Widrow et al. [1]. This problem is often considered a multifaceted one, for the interest can be placed either on estimating the frequency of the signal, on obtaining an enhanced version of it, or on tracking possible variations with time of the sinusoids frequencies. In any case, the application of such filters extends over a large number of fields. Some examples are: analysis of radar signals, removal of sinusoidal interference in biomedical or control applications [2,3], cancellation of periodic interference from signal measurements, tracking of a tone with time-varying frequency in communications systems [4,5], etc.

Our design basically derives from [6], with a generalization for a higher-order case using the cascade form and improved tracking capabilities by estimating both the central frequency and the bandwidth of the notch filter as in [7]. We also incorporate correction mechanisms for resolving closely spaced sinusoids and for dealing with very low or very high frequencies. This scheme is based on frequency transformations for digital filters and for this reason we call it the frequency-transform based notch filter (FTBNF).

\section{FREQUENCY-TRANSFORM BASED NOTCH FILTER}

By using the same transformation presented in [8] that converts a low-pass filter into a band-elimination filter, a new parametrization for a notch filter is introduced. These transformations present several advantages such as using all-pass structures, preserving spectral features after transformation and simplicity of implementation. The adaptation is accomplished by using the well-known Recursive Prediction Error (RPE) algorithm with the incorporation of several features, including an adaptive time-varying notch bandwidth and forgetting factor.

\section{A) Filter Structure Derivation}

The artifice consists in starting with a simple filter structure, which after a suitable transformation can be tuned to the input sinusoid frequency. Consider the $1^{\text {st }}$ order notch filter-a low-pass filter-given by

$$
H_{0}(z)=\frac{1+z^{-1}}{1+\rho z^{-1}}
$$

This filter is best called a notch filter with its central frequency located at $\omega_{0}-\pi$. As it is, the filter in (1) is not suitable for our problem since the only parameter we can modify is $\rho$ (the filter bandwidth). If we now apply the low-pass-to-band-elimination transformation given in [8], we have

$$
\begin{aligned}
H(z, \alpha) & =\frac{1+z^{-1} \frac{z^{-1}-\alpha}{1-\alpha z^{-1}}}{1+\rho z^{-1} \frac{z^{-1}-\alpha}{1-\alpha z^{-1}}} \\
& =\frac{1-2 \alpha z^{-1}+z^{-2}}{1-(1+\rho) \alpha z^{-1}+\rho z^{-2}}
\end{aligned}
$$

\footnotetext{
${ }^{+}$This work supported in part by the National Science Foundation under grant BCS-9308925
} 
This transformed filter has its zeros on the unit circle at $z_{0}=e^{ \pm / \omega_{0}}$, where $\omega_{0}$ satisfies the relationship $\omega_{0}=\cos ^{-1} \alpha$. In addition, for $\rho \rightarrow 1$ the poles are located at $z_{p} \approx \rho e^{ \pm j \omega_{0}}$. This is on the same radial line as the zeros but slightly inside the unit circle.

In order to ensure that the filter has complex conjugate poles, as required by the notch filter structure, we must add another constraint. This limitation restricts the range of frequencies the adaptive notch filter is able to remove. Specifically, by examining the denominator of (2) the constraint becomes

$$
\alpha^{2}<\frac{4 \rho}{(1+\rho)^{2}}
$$

The direct-form parameterization for the notch filter given by (2) may be useful to analyze certain filter characteristics, such as the bandwidth or the zero and pole location. Still, we are going to work with the structure suggested by the transformation shown in Figure 1 .

\section{B) Algorithm Derivation}

Recursive algorithms were extensively studied in the literature and good approaches to this topic can be found in [9-11]. For the FTBNF, we are going to implement the well-known RPE algorithm with some modifications to improve its performance. A very important element of every adaptive algorithm is the gradient of the error signal. Let us consider

$$
H_{n}(z)=\frac{B_{n}(z)}{A_{n}(z)}
$$

with $B_{n}(z)=1+z^{-1} H_{a p}(z)$ and $A_{n}(z)=1+\rho z^{-1} H_{a p}(z)$. Now, using the shift operator $q^{-1}$ defined by $q^{-n}[x(t)]=x(t-n)$ with integer $n$, we can rewrite (4) in the time domain as $A\left(q^{-1}\right) e(t)=B\left(q^{-1}\right) y(t)$. Then, using some assumptions from [10], the error gradient becomes

$$
\begin{aligned}
\varphi(t) & =\frac{\partial e(t)}{\partial \alpha} \\
& \approx \frac{1}{A_{n}\left(q^{-1}\right)}\left[\frac{\partial B_{n}\left(q^{-1}\right)}{\partial \alpha} y(t)-\frac{\partial A_{n}\left(q^{-1}\right)}{\partial \alpha} e(t)\right]
\end{aligned}
$$

where the adaptive parameter is $\alpha$ and all derivatives are taken with $\alpha=\alpha(t-1)$. Then

$$
\varphi(t) \approx \frac{1}{A_{n}\left(q^{-1}\right)} q^{-1} \frac{\partial H_{\alpha p}\left(q^{-1}\right)}{\partial \alpha}[y(t)-\rho e(t)]
$$

In order to simplify the algorithm further, we can use the gradient truncation given by

$$
\frac{\partial H\left(q^{-1}\right)}{\partial \theta}=\frac{\partial}{\partial \theta} \frac{A\left(q^{-1}\right)}{A\left(\rho q^{-1}\right)} \approx \frac{1}{A\left(\rho q^{-1}\right)} \frac{\partial A\left(q^{-1}\right)}{\partial \theta}
$$

This simplification leads to the following expression for the gradient

$$
\varphi(t) \approx \frac{\rho e(t-1)-y(t-1)}{1-(1+\rho) \alpha q^{-1}+\rho q^{-2}}
$$

It has been shown that the accuracy of the RPE algorithm when dealing with ANFs depends on both the forgetting factor of the estimation algorithm $(\lambda)$ and the pole contraction factor of the notch filter $(\rho)[7,12]$. In addition, the parameter $\rho$ plays a very important role when tracking non-stationary signals. The closer $\rho$ is to one, the longer the transients are, and the slower the tracking of rapidly changing signals becomes. Therefore, we need a mechanism to vary $\rho$ according to the input signal dynamics. We incorporate the adaptive notch bandwidth technique of [7]. This new adaptation is decoupled from the minimization of the all-pass filter parameter. Then, following the same analysis performed before, we have

$$
\begin{aligned}
\boldsymbol{\varphi}_{p}(t) & =\frac{\partial e(t)}{\partial \rho} \\
& \approx \frac{\alpha e(t-1)-e(t-2)}{1-(1+\rho) \alpha q^{-1}+\rho q^{-2}}
\end{aligned}
$$

\section{C) Multiple Sinusoid Case}

The extension of the single-sinusoid case to the more complex situation of having several sinusoids is not straightforward and can be approached from different perspectives. One such scheme uses the already described $2^{\text {nd }}$-order blocks and the convenient cascade form for digital filters as shown in Figure 2. Dealing with several sinusoids introduces some heretofore unconsidered issues. The change of the error surface into a multi-modal function is going to be one of the issues created by the increased problem dimension. Other obstacles are the resolution of closely spaced sinusoids, the tracking of signals with different dynamics, and the increase in required computational efforts. When tracking multiple sinusoids, the cascade structure of Figure 2 is recommended [13]. When $\rho$ is close to one, the bandwidths of each section do not overlap and each section in Figure 2 can effectively eliminate one sinusoid. So, instead of using the overall output signal to adjust the filter coefficients, this frequency decoupling property enables each section to be independently minimized. This becomes especially important when the frequencies in the input signal are time varying and follow different dynamics.

The advantages offered by the cascade configuration are manifold, and so we do not consider trying to generalize one of the approaches discussed in [14] in this paper. We note that this extended filter inherits all the properties of the one previously discussed such as stability monitoring, unimodality of the search surface, gradient simplicity and algorithm structure. 


\section{PERFORMANCE EVALUATION AND COMPARISON}

We present now the results of a simulation study of the proposed ANF. The simulations are programmed in $M^{M T L A B}{ }^{\text {TM }}$ and run on a PC. The examples presented here illustrate the behavior of the FTBNF under several conditions and emphasize the advantages of this new design. Parameters such as signal-to-noise ratio (SNR), number of sinusoids in the input signal $(n)$, number of samples in the signal $(N)$, and dynamics of the sinusoid frequencies, were used to simulate the FTBNF response under several operation environments.

The general input signal has the form

$$
y(t)=\sum_{i=1}^{n} C_{i} \sin \left[\pi \varphi_{i}(t)+\phi_{i}\right]+n(t), t=1,2, \ldots, N
$$

where $n(t)$ is the white Gaussian noise sequence with zero mean and variance $\sigma^{2} ; \phi_{i}$ is a random initial phase uniformly distributed in the interval $[0,2 \pi)$, and $\varphi_{i}(t)$ is the normalized phase for the $i$-th sinusoid. A normalized sampling frequency of $2 \mathrm{~Hz}$ is assumed throughout. The $S N R$ for each sinusoid is defined as $S N R_{i}=\frac{C_{i}^{2}}{2 \sigma^{2}}$, where $C_{i}$ is the amplitude of the $i$-th sinusoid and $\sigma^{2}$ the noise power. We are interested in evaluating the following features:

- Algorithm convergence. When choosing $\rho$ it is important to consider three points; the effect in the notch bandwidth, the shape of the error performance surface, and the range of reachable frequencies obtained by applying the constraint in (3) and using the relationship $\alpha=\cos \omega$.

- Estimation accuracy and operation range. A plot of the true frequency vs. the estimated frequency is presented in Figure 3 to validate our affirmation of accuracy.

- Filter tracking capabilities for different signal dynamics.

- Algorithm convergence speed. The speed of convergence is measured by computing the time $t_{c}$ such that

$\left|\hat{f}_{i}(t)-f_{i}\right|<0.05^{*} f_{i}$ for $t \geq t_{c} \forall i=1,2, \ldots, n$

The accuracy when the filter converges to the correct set of parameters is intimately related to the statistical analysis.

- Statistical properties such as the bias and standard deviation of the estimated sinusoid frequencies.

- Computational complexity given by the algorithm efficiency vs. simplicity and structure impact for the gradient computation and stability monitoring.

We are only able to show the most relevant results in [14].

\section{Simulation Results}

We analyzed the tracking abilities of our algorithm when dealing with multiple sinusoids each of them having different dynamics. The plot in Figure 4 depicts the behavior of the FTBNF showing the evolution with time of the estimated frequency $(f)$ and the constraint factor $(\rho)$. This simulation shows the superior tracking capabilities of the FTBNF when compared to similar designs. While one frequency is modulated with a ramp function (chirp signal), the other one varies as a sine wave around $f=0.4$ $\mathrm{Hz}$ (FM signal), and the last one varies its frequency with the previously used step function, from $f=0.2 \mathrm{~Hz}$ to $f=0.15$ $\mathrm{Hz}$ at $t=500$. The FTBNF accurately follows the three signals instantaneous variations.

\section{CONCLUSIONS}

We addressed the problem of designing a fully adaptive notch filter for detecting and tracking multiple nonstationary sinusoid signals immersed in additive white noise. We discussed the use of cascade structures and the frequency transformations for digital filters that are given by all-pass filters substituting for the basic delay operator. The design and performance evaluation proved the new filter suitability for its application over a large number of situations. However, because of space restrictions a good number of simulations could not be reported here. For a more detailed analysis see [14].

The resulting notch filter transfer function has its zeros on the unit circle and the poles on approximately the same radial line, but slightly inside the unit circle to ensure the filter stability. The adaptive parameters consist of the allpass filter coefficients and the corresponding pole contractor factor for each section. The use of an $a$ posteriori prediction error improves the algorithm convergence. On the other hand, gradient truncation is feasible and efficient due to the explicit use of all-pass filters in the notch filter transfer function. Performance for non-stationary signals was improved by making the parameter $\rho$ adaptive. In this way, the notch bandwidth broadens when frequency variations are detected, and narrows after convergence has been achieved. Summarizing, we can see that the FTBNF is the adequate choice for non-stationary environments, where no a priori information on the signals is provided. This new scheme successfully tracks different signal dynamics under a wide variety of conditions. In addition, the convergence time, statistical performance and computational complexity are not sacrificed to a great extent as proved in [14].

\section{REFERENCES}

[1] P. A. Thompson, "A constrained recursive adaptive filter for enhancement of narrowband signals in white noise," in Proc. 12th Asilomar Conf. Circuits, Syst. Comput., Pacific Grove, CA, Nov. 1978, pp. 214-218. 
[2] S.C. Pei and C.C. Tseng, "Complex adaptive IIR notch filter algorithm and its applications," IEEE Trans. Circuits Syst.II, vol. 41, no. 2, pp. 158-163, Feb. 1994.

[3] M. Ferdjallah and R.E. Barr, "Adaptive digital notch filter on the unit circle for the removal of powerline noise from biomedical signals," IEEE Trans. Biomed. Eng., vol. BME-41, no. 6, pp. 529-536, June 1994.

[4] D.R. Hush, N. Ahmed, R. David and S.D. Stearns, "An adaptive IIR structure for sinusoidal enhancement, frequency estimation, and detection," IEEE Trans. on Acoust., Speech, Signal Processing, vol. ASSP-34, no. 6, pp. 1380-1389, Dec. 1986.

[5] S.C. Pei and C.C. Tseng, "Real time cascade adaptive notch filter scheme for sinusoidal parameter estimation," Sig. Proc., no. 39, pp. 117-130, 1994.

[6] J.A. Chambers, "Frequency tracking using constrained adaptive notch filters synthesized from allpass sections," Proc. Inst. Elect. Eng., Dec. 1990, vol. 137, Pt. F, no. 6, pp. 475-481.

[7] M.V. Dragoševic and S.S. Stankovic, "Fully adaptive constrained notch filter for tracking multiple frequencies," Electron. Lett., vol. 31, no. 15, pp. 1215-1217, July 1995.

[8] A. Constantinides, "Spectral transformations for digital filters," Proc. IEEE, vol. 117, no. 8, pp. 1585 1590, Aug. 1970.

[9] L. Ljung and T. Söderström, Theory and Practice of Recursive Identification. Cambridge, MA: MIT Press, 1983.

[10]J.J. Shynk, "Adaptive IIR Filtering," IEEE ASSP Magazine, pp.421, Apr. 1989.

[11]T. Söderström, L. Ljung and I. Gustavsson, "A theoretical analysis of recursive identification methods," Automatica, vol. 14, pp. 231-244, 1978.

[12]M.V. Dragoševic and S.S. Stankovic, "An adaptive notch filter with improved tracking properties," IEEE Trans. Signal Processing, vol. 43, no. 9, pp. 20682077, Sep. 1995.

[13]T. Kwan and K. Martin, "Adaptive detection and enhancement of multiple sinusoids using a cascade IIR filter," IEEE Trans. Circuits Syst., vol. 36, no. 7, pp. 937-947, July 1989.

[14] S.M. Torres, "Analysis, comparison and design of an adaptive digital notch filter," M.S. thesis, The University of Oklahoma, Norman, OK, 1997.

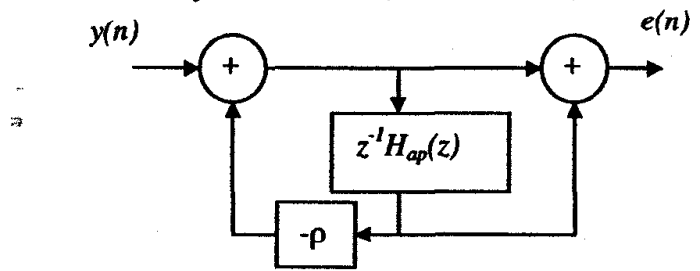

Figure 1. Notch filter parametrization. The scheme corresponds to a transformed notch filter at $\omega_{0}=\cos ^{-1} \alpha$.

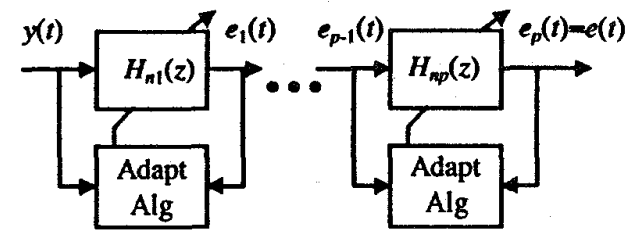

Figure 2. Cascade form for the FTBNF. Each section is formed with a $2^{\text {nd }}$-order FTBNF and the corresponding independent adaptive algorithm.

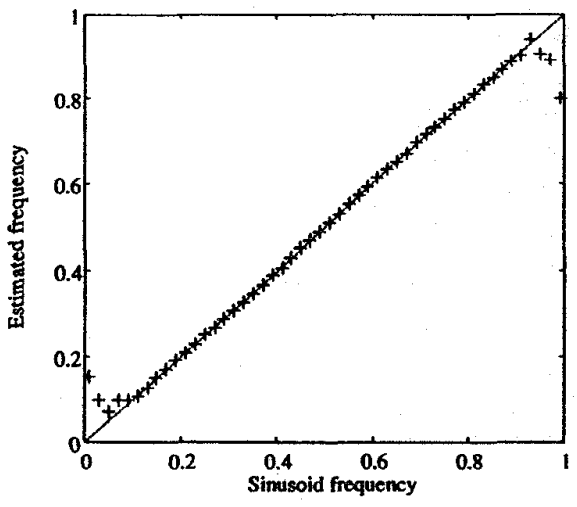

Figure 3. Estimated frequency vs. true sinusoid frequency. The symbol + represents the averaged estimated frequency for 10 experiments. The signal has the form $y(t)-A \sin \left(\pi f_{n} t\right)$ $+n(t)$, with $S N R=10 \mathrm{~dB}$.
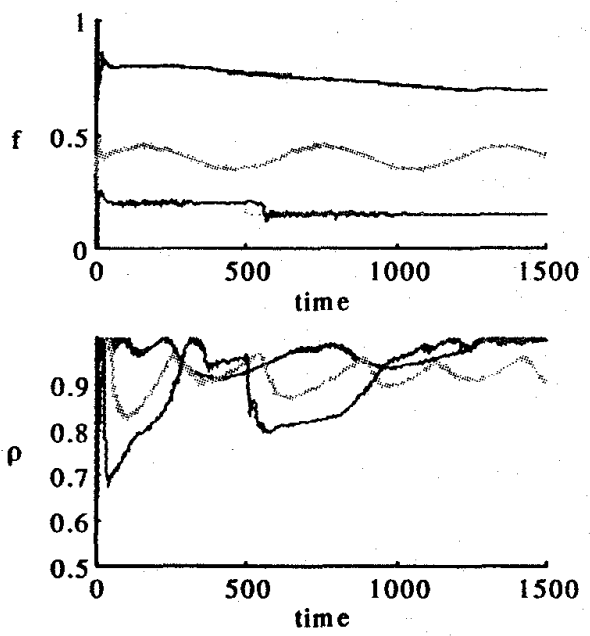

Figure 4. Simulation with non-stationary multiple sinusoids using signals with different dynamics. A chirp signal, an FM signal and frequency jump signal at an SNR of $10 \mathrm{~dB}$ for each component were combined to generate the combined signal. 